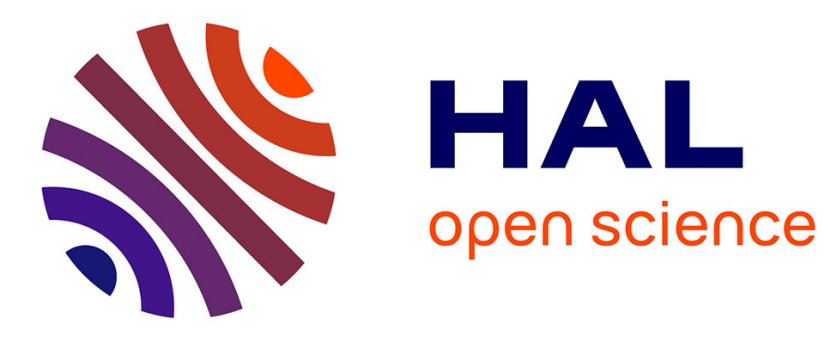

\title{
Effect of the point mutation H148G on GFPmut2 unfolding kinetics by fluorescence spectrocopy
}

Chiara Bosisio, Valentina Quercioli, Giuseppe Chirico, Laura d'Alfonso, Stefano Bettati, Samanta Raboni, Barbara Campanini, Maddalena Collini

\section{- To cite this version:}

Chiara Bosisio, Valentina Quercioli, Giuseppe Chirico, Laura d'Alfonso, Stefano Bettati, et al.. Effect of the point mutation H148G on GFPmut2 unfolding kinetics by fluorescence spectrocopy. Biophysical Chemistry, 2011, 10.1016/j.bpc.2011.04.004 . hal-00759071

\section{HAL Id: hal-00759071 https://hal.science/hal-00759071}

Submitted on 30 Nov 2012

HAL is a multi-disciplinary open access archive for the deposit and dissemination of scientific research documents, whether they are published or not. The documents may come from teaching and research institutions in France or abroad, or from public or private research centers.
L'archive ouverte pluridisciplinaire HAL, est destinée au dépôt et à la diffusion de documents scientifiques de niveau recherche, publiés ou non, émanant des établissements d'enseignement et de recherche français ou étrangers, des laboratoires publics ou privés. 


\section{Accepted Manuscript}

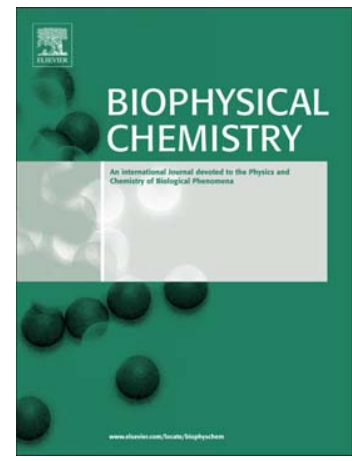

Effect of the point mutation H148G on GFPmut2 unfolding kinetics by fluorescence spectrocopy

Chiara Bosisio, Valentina Quercioli, Giuseppe Chirico, Laura D'Alfonso, Stefano Bettati, Samanta Raboni, Barbara Campanini, Maddalena Collini

PII:

S0301-4622(11)00073-1

DOI:

doi: 10.1016/j.bpc.2011.04.004

Reference:

BIOCHE 5500

To appear in: Biophysical Chemistry

Received date: 1 March 2011

Revised date: $\quad 4$ April 2011

Accepted date: $\quad 4$ April 2011

Please cite this article as: Chiara Bosisio, Valentina Quercioli, Giuseppe Chirico, Laura D'Alfonso, Stefano Bettati, Samanta Raboni, Barbara Campanini, Maddalena Collini, Effect of the point mutation H148G on GFPmut2 unfolding kinetics by fluorescence spectrocopy, Biophysical Chemistry (2011), doi: 10.1016/j.bpc.2011.04.004

This is a PDF file of an unedited manuscript that has been accepted for publication. As a service to our customers we are providing this early version of the manuscript. The manuscript will undergo copyediting, typesetting, and review of the resulting proof before it is published in its final form. Please note that during the production process errors may be discovered which could affect the content, and all legal disclaimers that apply to the journal pertain. 


\section{Effect of the point mutation H148G on GFPmut2 unfolding kinetics by fluorescence spectrocopy.}

Chiara Bosisio ${ }^{a}$, Valentina Quercioli ${ }^{d}$, Giuseppe Chirico ${ }^{a}$, Laura D'Alfonso $^{a}$, Stefano Bettati $^{b, c}$, Samanta Raboni ${ }^{b}$, Barbara Campanini ${ }^{b}$ and Maddalena Collini ${ }^{a, *}$

${ }^{a}$ Dipartimento di Fisica, Università degli Studi di Milano-Bicocca, Piazza della Scienza 3, 20126 Milano, Italy.

${ }^{b}$ Dipartimento di Biochimica e Biologia Molecolare, Università degli Studi di Parma, Viale Usberti 23/A, 43124 Parma, Italy

${ }^{c}$ Istituto Nazionale di Biostrutture e Biosistemi, Rom, Italy.

${ }^{d}$ Laboratorio di Biologia Molecolare, Scuola Normale Superiore, Pisa, 50126

*Dipartimento di Fisica, Università degli Studi di Milano-Bicocca, Piazza della Scienza 3, 20126 Milano, Italy, voice: +390264482437 fax: +390264482585 email:maddalena.collini@mib.infn.it 


\begin{abstract}
We have used fluorescence spectroscopy techniques such as fluorescence correlation spectroscopy and fluorescence anisotropy decay on a wide time range, from nanoseconds to seconds, to investigate the unfolding kinetics induced by guanidinium chloride of GFPMut2 and its point mutation $\mathrm{H} 148 \mathrm{G}$, which has proved to be relevant for GFP photochemistry and photophysics.

The mutation affects the unfolding kinetics of GFP leading to a much faster process at alkaline $\mathrm{pH}$ values, where protonation dynamics is negligible, that can be ascribed to a twofold role of His148, either as a proton shutter towards the chromophore and as a conformation stabiliser. For both mutants a soft region located near beta-strand 3 is found that starts to gain flexibility in the ns range at denaturant concentrations far lower than those required to turn off the chromophore fluorescence, as derived from the anisotropy decay of an extrinsic probe covalently bound to the proteins.
\end{abstract}

Keywords: GFP, unfolding, fluorescence anisotropy, fluorescence correlation spectroscopy, (H148G, GFPmut2).

1 


\section{INTRODUCTION}

Since its widespread use as a cellular reporter (1), either alone as a fluorescent marker in vivo or as a chimeric construct (2), a better and deeper understanding of the unfolding kinetics of the Green Fluorescent Protein (GFP) from Aequorea Victoria is relevant both for basic and applied research where its mutants are used for subcellular localizations. It has been shown (3) that protein structure, and in particular the beta-barrel scaffold, is determinant in ensuring a high fluorescence quantum yield by maintaining a semi-rigid environment around the chromophore because this low conformational flexibility should reduce the non radiative de-excitation pathways. However, some degree of structure flexibility should be welcome in order to allow those structural transitions, such as cistrans isomerizations, protonations, proton transfers (4), that can lead to photoswitchable probes. The design of GFP mutants with different photodynamics properties is in fact desirable in view to be exploited for the most recent super-resolution applications of fluorescence microscopy $(5,6)$.

A good indicator of the integrity of the protein scaffold, which is mainly due to the 11 strands beta barrel structure comes from the fluorescence of the chromophore, which is quenched when it is exposed to the solvent, as suggested by the correlation between the barrel structure detected by far UV circular dichroism and by the green fluorescence (7). GFP unfolding kinetics is extremely slow, reaching equilibrium after hours or days, depending on the mutant and on the denaturant concentration. In the literature, a number of works are found on the unfolding features of cycle3 mutant (8-13) which shows stable intermediates upon both acidic and chemical denaturation, and reaches equilibrium on weeks time-scale. A certain degree of irreversibility in the refolding process has been reported (8), described by double exponential reaction curves and ascribed, at least partially, to proline residues isomerization. Comparative studies on other fluorescent proteins $(3,12,14-17)$ show that all the FPs share uncommonly long unfolding kinetics. As suggested in a recent review (3), a rough energy landscape with high energy barriers (18-23) is likely to characterize the GFP unfolding pathway.

NMR studies (24) has suggested that the mutation of histidine 148 with a glycine in strand 7 could alter the backbone dynamics affecting the protein stability. Since our group has recently characterized the photodynamic effects of such mutation on GFP (25), 
it is particularly interesting to investigate the coupling between the structural flexibility and the photodynamics whose understanding seems to be relevant for the development of photochromic GFPs.

In the present paper we investigate the unfolding kinetics of this point mutation H148G of GFPmut2 (S65A/V68L/S72A GFP) compared to the parent protein in the presence of guanidinium chloride $(\mathrm{GuHCl})$. GFPmut2 (Mut2) is a GFP folding mutant optimized for live cell spectroscopy due to high expression yield in bacteria, high fluorescent yield (26) and increased $\mathrm{pKa}$ for the transition between the protonated and the highly fluorescent anionic form of the chromophore (27). However, its unfolding kinetics at $\mathrm{pH} 7$ is still as slow as that of the wild type protein (7). The H148G mutation on Mut2, yielding Mut2G, has a large effect on the chromophore $\mathrm{pKa}$, which is shifted from $\sim 6.0$ to $\sim 7.5(25,28)$ and on the chromophore protonation kinetics $(25)$, yielding an increase of the protonation rate due to the missing proton shutter, the histidine, between the solvent and the chromophore.

Different techniques such as fluorescence lifetime decay, fluorescence correlation spectroscopy (FCS) and fluorescence anisotropy decay (FPA) have been combined in order to gain insights in the possible conformational changes accompanying the unfolding transition and the coupling of conformational changes to photodynamics with a particular attention to alkaline $\mathrm{pH}$, where the protein is highly fluorescent. Since the fluorescence of the chromophore ceases when it is exposed to the solvent, we also exploited an extrinsic fluorescent probe, tetra-methyl-rhodamine (TMR), bound to the free $\mathrm{SH}$ group of Cys48 (beta-sheet 3) in order to detect conformational changes when the chromophore is already quenched.

\section{EXPERIMENTAL PROCEDURES}

Protein. Mut2 and Mut2G were expressed and purified as described elsewhere (28). GFP mutants were diluted in Tris- $\mathrm{NaCl}$ or acetate buffers at $\mathrm{pH}=8.4 \pm 0.2$, and GuHCl (Sigma G9284, Guanidine hydrochloride solution, $8 \mathrm{M}$ ) was added to obtain the desired denaturant concentration. For experiments with the extrinsic fluorescent probe, GFP was derivatized with tetramethylrhodamine-5-maleimide (Fluka 94506) on Cys48. 
The labeling degree, as verified by absorption spectroscopy measurements, was about $30 \%$.

Protein concentration was 10-100 nM for fluorescence kinetics, lifetime and FCS experiments, and $0.5-1 \mu \mathrm{M}$ for FPA measurements.

Kinetic Fluorescence Measurements. The unfolding reactions were initiated by manual mixing of protein and denaturant solutions. The kinetics have been followed in the time range of 2-200 minutes monitoring the fluorescence signal. All experiments were carried out at $22 \pm 0.5^{\circ} \mathrm{C}$.

Microspectrometer Setup. A home assembled micro-spectrometer based on an inverted TE 300 Nikon microscope has been used for fluorescence experiments at nanomolar concentrations, lifetime decay in the time domain and FCS. The two-photon excitation (TPE) source was based on a pulsed Ti:sapphire laser (Tsunami 3960, Spectra Physics, CA) coupled to the microscope. The laser provides $280 \mathrm{fs}$ pulses on the sample plane at a repetition frequency of $80 \mathrm{MHz}$ in the range of 700-1000 nm. GFP mutants have been excited at $890 \mathrm{~nm}$. The fluorescence signal, collected by a Plan Apochromat $60 \times$ water objective (NA 1.2, Nikon, Japan), is separated from the excitation beam by a dichroic mirror and further selected by an emission band-pass filter (HQ515/30 or HQ570/30, Chroma Inc., Brattelboro, VT). The fluorescence signal is split by a nonpolarizing 50\% cube splitter and fed directly to two single-photon avalanche diode (SPAD) modules (Perkin-Elmer, Canada; model SPCM-AQR15). The minimum values of the radial and axial fwhm of the microscope point spread function (PSF) are $240 \pm 40$ $\mathrm{nm}$ and $780 \pm 50 \mathrm{~nm}$, respectively, at a wavelength of $800 \mathrm{~nm}(28)$. The excitation volume employed in the following experiments was about $0.85 \mu \mathrm{m}^{3}$. Fluorescence spectra of samples on the microscope stage were acquired by means of a CCD (DV420A-BV, Andor, IRL)-based spectrometer (MS125, Lot-Oriel, U.K.).

Fluorescence Correlation Functions. The autocorrelation functions (ACFs) were acquired by an ALV5000E (ALV, Langen, D) board and analysed by means of the non least- squares routine of the Origin 7.0 software (OriginLab Inc., Northampton, MA). Actually, since the fluorescence signal can be split on two SPADs, cross correlation detection is used in order to avoid detector dead time and afterpulsing that limit the resolution of decay times in the microseconds region. However, since the two SPADs 
detect the same fluorescence signal coming from the same volume, the pseudo-cross correlation function can be identified with an autocorrelation function where the detectors "defects" have been cleared out: therefore, in order to simplify the notation in the following, the term ACF will still be used to indicate both the fitting expressions and the experimental curves.

In general, the ACFs were fit to an expression taking into account both the diffusive decay of the ACF and the internal photodynamic part of the time dependence:

$$
G(t)=G(0)\left(1+\frac{t}{\tau_{D}}\right)^{-1}\left(1+\left(\frac{\lambda}{\sqrt{2} \pi \omega_{0}}\right)^{2} \frac{t}{\tau_{D}}\right)^{-0.5} \times \prod_{j}\left[1+\frac{A_{j}}{1+A_{j}} \exp \left(-\frac{t}{\tau_{j}}\right)\right]
$$

with $\mathrm{G}(0)=\gamma /\langle\mathrm{N}\rangle$, where $\gamma=0.076$ for TPE is a geometrical factor, $\langle\mathrm{N}\rangle$ is the average number of protein in the excitation volume, $\omega_{0}$ is the beam waist, $\lambda=890 \mathrm{~nm}, \tau_{\mathrm{D}}=\omega_{0}{ }^{2} / 8 \mathrm{D}$ is the diffusional time of GFP with a translational diffusion coefficient $D, \tau_{j}$ is the $j$-th photodynamic characteristic time and $\mathrm{A}_{\mathrm{j}}$ is the protein fraction in the $\mathrm{j}$-th dark state.

Fluorescence Lifetimes in Time Domain. A PCI board for time-correlated singlephoton counting (TCSPC) (Time Harp 200, PicoQuant, Berlin, D) was employed to measure the times of arrival (with respect to the laser pulse) of photons collected from protein solutions by the SPAD module. The full half-height width of the intensity response function is about $350 \mathrm{ps}$.

In general, the fluorescence lifetime decay can be analysed as a sum of discrete exponential components

$$
I(t)=\sum_{i} \alpha_{i} \exp \left(-t / \tau_{i}\right) \quad \mathrm{f}_{i}=\alpha_{i} \tau_{i} / \sum_{i} \alpha_{i} \tau_{i}
$$

where $\tau_{\mathrm{i}}$ are the lifetime values with amplitudes $\alpha_{\mathrm{i}}$ and fractional intensities $\mathrm{f}_{\mathrm{i}}$.

Fluorescence Anisotropy Kinetics in the Frequency Domain. Dynamic fluorescence measurements were performed on a frequency modulated phase fluorometer (Digital K2, ISS, Champaign, IL). The excitation of GFP and of the TMR dye was primed by the 488-nm or the 514-nm emission lines of an argon ion laser (Spectra Physics, 2025, Mountain View, CA). The laser emission was modulated by a Pockels cell over a frequency range of $10-150 \mathrm{MHz}$ and the data acquired through a digital data 
acquisition board at $400 \mathrm{~Hz}$ cross-correlation frequency (A2D, ISS, Champaign, IL). The accuracy of the phase angles and modulation ratios was at least $0.2^{\circ}$ and 0.004 , respectively. The Rayleigh and Raman scattering were cut by band-pass filters that select the sample emission at 515/30 nm (for GFP) and at 560/40 $\mathrm{nm}$ for TMR and the sample was kept at $22 \pm 0.2^{\circ} \mathrm{C}$. For further details, see Collini et al. (30). For the lifetime measurements, the emission polarizer was set at the magic angle position (31). The dependence of the phase shift and the demodulation ratio of the fluorescence signal was measured with respect to the excitation beam as a function of the modulation frequency and can be computed from the Laplace transform L(I) of the intensity decay:

$$
L(I)(\omega)=m(\omega) \exp [i \phi(\omega)]
$$

where $m(\omega)$ and $\phi(\omega)$ are the modulation ratio and the phase shift of the fluorescence output with respect to the reference one. If a multi-exponential function describes the decay as in Eq.2, the fit of phase shifts and demodulation ratios versus modulation frequency to Eq.4 with $L(I)(\omega)=\sum_{i} f_{i} /\left(1+i \omega \tau_{i}\right)$, gives the parameters of the fluorescence decay $\tau_{\mathrm{i}}$ and $\mathrm{f}_{\mathrm{i}}$.

When performing a fluorescence anisotropy decay measurement, the excitation beam is vertically polarized and the emission is detected both in the parallel $\mathrm{I}_{\|}$and in the perpendicular $\mathrm{I}_{\perp}$ polarizer configuration. The anisotropy $\mathrm{r}(\mathrm{t})$ is related to the rotational correlation time of the fluorophore by:

$$
r(t)=\frac{I_{\|}-I_{\perp}}{I_{\|}+2 I_{\perp}}=r_{0} \sum_{j} g_{j} \exp \left(-t / \theta_{j}\right)
$$

where $r_{0}$ is the limiting anisotropy, $\theta_{\mathrm{j}}$ are the rotational correlation times with relative amplitudes $g_{\mathrm{j}}$ normalised to unity. In the frequency domain we measure the difference in phase angles $\Delta_{\omega}$ and the demodulation ratios $\Lambda_{\omega}$ of the perpendicular component with respect to the parallel one:

$$
\Delta_{\omega}=\phi_{\perp}(\omega)-\phi_{\|}(\omega) \quad \Lambda_{\omega}=\mathrm{M}_{\perp}(\omega) / \mathrm{M}_{\|}(\omega)
$$


These are related to the intensity components, according to (28):

$$
L\left(I_{\|}\right)(\omega) / L\left(I_{\perp}\right)(\omega)=\Lambda_{\omega} \exp \left(i \Delta_{\omega}\right)
$$

where $\mathrm{L}\left(\mathrm{I}_{\perp, \|}\right)(\omega)$ are the Laplace transforms of the perpendicular and parallel components of the fluorescence intensity. In this work we have always found that the time decay of $r(t)$ can be fitted by the sum of two exponentials, where $\theta_{1}$ and $\theta_{2}$ are the rotational correlation times with relative amplitudes $g_{1}$ and $g_{2}=1-g_{1}$. All fits were performed by VINCI-Analysis (ISS, Champaign, IL) software.

\section{RESULTS}

Our aim has been to bring into evidence the fluorescence fluctuations related to the protein structure destabilization during the unfolding process in Mut2 and in its single point mutation Mut2G. Since the protonation equilibrium plays a leading role at $\mathrm{pH}$ values close or lower than the protein $\mathrm{pK}$, we chose an alkaline $\mathrm{pH}$ value of 8.4 where protonation is negligible and the proteins reside in their anionic highly fluorescent state.

GFP Unfolding Kinetics. In order to determine the appropriate denaturant concentrations and time range for the FCS experiments, the unfolding kinetics of Mut2 and Mut2G have been monitored at nanomolar protein concentration by manual mixing of the guanidinium chloride $(\mathrm{GuHCl})$ and protein solutions at alkaline $(\mathrm{pH}=8.4) \mathrm{pH}$ values by steady-state fluorescence. The equilibrium unfolding curves have been measured by acquiring the fluorescence signal several hours after $\mathrm{GuHCl}$ addition (Fig.1A). The curves have been described by a two-state model (continuous lines) obtaining a midpoint $\mathrm{GuHCl}$ concentration of $2.4 \pm 0.2 \mathrm{M}$ and $1.1 \pm 0.1 \mathrm{M}$ for Mut2 and Mut2G, respectively, thus ensuring that at $\mathrm{GuHCl}$ concentrations larger than $3 \mathrm{M}$ the unfolding rate dominates the reaction. Due to the lack of full reversibility (7) as confirmed by the slow relaxations in equilibrium measurements (13), the curves in Fig.1A should be considered as pseudoequilibrium titrations and therefore we cannot derive meaningful values of the free energy gains involved in the unfolding process.

The unfolding kinetics have been obtained either by the fluorescence signal at $510 \mathrm{~nm}$ (the peak wavelength of the anionic emission (25)), or by acquiring the whole spectrum 
with a CCD camera spectrometer (time resolution $=1 \mathrm{~s}$ ). In Figure 1 the kinetics profiles are shown for different $\mathrm{GuHCl}$ concentrations for Mut2 (panel B) and Mut2G (panel C). The data can be described by a double exponential decay whose longer relaxation time,

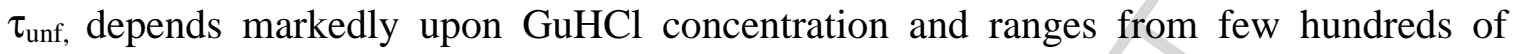
seconds (close to the lower limit of manual mixing of our setup, $\approx 120 \mathrm{~s}$ ) to thousands of seconds. This component can be related to the apparent kinetic rate constant for the unfolding process, $\mathrm{k}_{\mathrm{obs}}=1 / \tau_{\mathrm{unf}}$, that has a linear dependence on the denaturant concentration, as shown in Figure 1D.

On the contrary the shorter component, whose relaxation time falls in the range of $200 \mathrm{~s}$, does not show any appreciable dependence upon the solution conditions, and it has been attributed to specific effects of chloride ions on GFP fluorescence $(7,32)$. A control experiment performed in the presence of $4 \mathrm{M} \mathrm{NaCl}$ has shown that the fluorescence intensity shows indeed a time dependence, with a characteristic decay time of about 200 $\pm 30 \mathrm{~s}$, as shown in Figure 2A. In particular, an intensity decrease of $15 \%$ and $25 \%$ is determined for Mut2 and Mut2G, respectively. $\mathrm{NaCl}$ is known to induce a significant decrease in the fluorescence emission of $\operatorname{GFP}(33,34)$ since it has a specific binding site (35), and similar findings have been reported also on other proteins (36). This fast fluorescence decay process should therefore not be treated as part of the unfolding process.

Fluorescence Lifetime. The value of the fluorescence lifetime during the unfolding kinetics can give information about changes in the chromophore environment during the action of the denaturant. The GFP chromophore lifetime values in the presence of $\mathrm{GuHCl}$ are systematically shorter than those obtained in the absence of denaturant. This effect can be however ascribed to the protein-chloride ions interaction, similarly to the behaviour observed for the fluorescence emission intensity (Fig.2A). In particular, measurements in the presence of $4 \mathrm{M} \mathrm{NaCl}$ show that the lifetime decreases by $15-20 \%$ for Mut2 and Mut2G. Apart from this unspecific effect, the excited state lifetime values, averaged over experiment durations much smaller than the unfolding characteristic time, $1 / \mathrm{k}_{\mathrm{obs}}$, remain constant for both mutants independent of $\mathrm{GuHCl}$ as shown for example in Figure $2 \mathrm{~B}$ at $[\mathrm{GuHCl}]=5.5 \mathrm{M}$ and $4.5 \mathrm{M}$ for Mut2 and Mut2G, respectively.

Quasi Static Fluorescence Correlation Spectroscopy. FCS autocorrelation 
functions $(\mathrm{ACFs})$ contain information on the brightness fluctuations due either to reactions at the excited state (photophysics) or at the ground state (chemical/ structural reactions). The ACFs profiles collected under unfolding conditions at $\mathrm{pH} 8.4$ are shown as a function of time in Figure 3A for Mut2 at 5.5 M GuHCl and in Figure 3B for Mut2G at $4.2 \mathrm{M} \mathrm{GuHCl}$ as continuous lines, whereas the corresponding reference ACFs in the absence of denaturant are shown as symbols. From the extrapolation at zero lag time, $\mathrm{G}(0)$, as obtained by fitting the profiles to Eq.(1), we can extract a measure of the average number of molecules $\langle\mathrm{N}\rangle$ in the excitation volume. $\mathrm{G}(0)$ does increase as the denaturation proceeds, corresponding to values of $\langle\mathrm{N}\rangle$ that decrease exponentially (Figure 3C, 3D) with characteristic time constants $\tau=3900 \pm 240 \mathrm{~s}$ for Mut2 and $\tau=$ $2700 \pm 120 \mathrm{~s}$ for Mut2G. A second relevant parameter, that can be estimated once $\mathrm{G}(0)$ is known, is the molecular brightness of the chromophore, computed as the ratio of the fluorescence rate to the number of molecules in the observation volume, $\varepsilon=\langle\mathrm{F}\rangle \mid\langle\mathrm{N}\rangle=$ $<\mathrm{F}>\mathrm{G}(0)$. Also this parameter appears to be constant during denaturation (insets of Figure $3 \mathrm{C}$ and $3 \mathrm{D})$.

From the time behaviour of the fluorescence correlation curves we can instead gain information on the protein structural and chemical dynamics. The diffusive component of the ACFs falls around $0.5-1 \mathrm{~ms}$, depending on the $\mathrm{GuHCl}$ concentration that affects the solution viscosity. This component is found constant within the experimental error in all the kinetics investigated, giving a translational diffusion coefficient $\mathrm{D} \cong 90 \pm 5 \mu \mathrm{m}^{2} / \mathrm{s}$ in the absence of $\mathrm{GuHCl}$ and ranging from 70 to $60 \mu \mathrm{m}^{2} / \mathrm{s}$ at $\mathrm{GuHCl}$ concentrations o $4.4 \mathrm{M}$ to $5.2 \mathrm{M}$. However, the translational diffusion coefficient is a parameter that is not very sensitive to protein volume changes, since it scales only as the inverse of the protein effective radius: volume changes within $10 \%$ give diffusion coefficient variations of about $3 \%$, which are often within the experimental uncertainty.

Under native conditions a single photodynamic component (Eq. 1) can be singled out with a characteristic time of about 100-250 microseconds. The fraction of proteins switching to this dark state, probably due to a cis-trans conformational transition is at most $20-25 \%$ at the power level used in the experiments, in agreement with a previous 
systematic study (25). In the presence of denaturant no additional components are brought into evidence in both mutants but a larger fraction (40-60\%) of this fast component is needed to fit the ACFs as shown in the inset of Fig.3B. This cannot be ascribed to $\mathrm{Cl}^{-}$ions specific effects as it has been checked by FCS measurements in the presence of $4 \mathrm{M} \mathrm{NaCl}$ (data not shown).

Fluorescence Polarisation Anisotropy Decay. Fluorescence polarisation anisotropy (FPA) decay is sensitive to motions in the nanosecond time range that is typical of the polymer internal motions (37). It is used here to obtain information complementary to those obtained by FCS, which is typically sensitive to fluctuations longer than $1 \mu \mathrm{s}$. In particular, due to the long unfolding kinetics of GFP, it is possible to follow changes in the fluorescence anisotropy decay of the two mutants as a function of time during the denaturation process.

In the absence of denaturant, the fluorescence anisotropy of the chromophore can be described by a single exponential decay for both mutants. The values of the correlation time, shown in Table 1, are close to $14 \mathrm{~ns}$ and are very similar for the two proteins, showing that the point mutation does not influence appreciably the overall encumbrance when monitored by the chromophore fluorescence.

The rotational correlation time can be related to the volume occupied by a spherical rotator through the well known Einstein relation, $\theta=\eta V / k T=4 \pi \eta R_{\text {eff }}^{3} / 3 k T$, where $\eta$ is the solution viscosity at temperature $T$, by which an effective radius $R_{\text {eff }}=2.4$ $\mathrm{nm}$ can be estimated. No faster components due to possible wobbling motions of the chromophore have been detected within the instrumental time resolution $(0.1 \mathrm{~ns})$. The measured effective radius of both mutants, substantially larger than that expected for an equivalent sphere of the same volume as inferred from the crystallographic data, about $1.75 \mathrm{~nm}$, is close to the value found for other mutants (38-40) when taking into account the barrel shape of the protein and the 60 degrees orientation of the chromophore with respect to the axis of the beta-barrel. Therefore, the samples examined are in the monomeric form.

When $\mathrm{GuHCl}$ is added to the solution up to $6.0 \mathrm{M}$ concentration, the rotational correlation time increase according to the increased solution viscosity, but the effective radius remains unchanged (Figure 2D). Also when the unfolding kinetics is followed 
versus time, the FPA decay time does not show any change, as shown in Figure $2 \mathrm{C}$. To be noted that no additional wobbling components appear in the FPA decay during the whole unfolding process.

TMR Fluorescence Polarization Anisotropy. In order to unveil possible intramolecular motions of the beta-barrel during the whole unfolding process, even after the chromophore has lost its fluorescence, we have monitored the fluorescence of an extrinsic probe, TMR, complexed to GFP. The dye was bound through a thioester bond to the cysteine in position 48, located at the end of the beta-strand 3. The position is optimal to detect hinge motions of the beta-barrel structure in a region that is not closely related to the chromophore environment. In order to ascertain whether TMR labelling at residue 48 could alter GFP stability, we have acquired far UV circular dichroism spectra versus denaturant concentration, shown in the inset of Figure 4B, at 0, 1M, 2M and 4M of $\mathrm{GuHCl}$ for Mut2.

TMR can be excited by the green line of an Argon laser and it emits at wavelengths longer than those of the chromophore (emission peak at $560 \mathrm{~nm}$ ).

In the absence of $\mathrm{GuHCl}$, the FPA measurements of the labelled protein give two correlation times for both mutants, contrary to the chromophore fluorescence case, as shown in Table 1. The longer correlation time, about $15 \mathrm{~ns}$, can be related to the overall protein rotation, while the shorter one, $\approx 1 \mathrm{~ns}$, is related to the libration (wobbling) motion of the probe at the binding site. As deduced from the relative weights, approximately one third of the depolarisation is accounted for by the probe motion. Due to the different time scale of the two motions and assuming that the segmental motion of the probe occur independently of the overall rotational motions, we can describe the FPA decay in terms of a hindered motion of the TMR probe. The FPA drops rapidly from the initial $\mathrm{r}_{0}$ value to a lower anisotropy value, $r_{\infty}=r_{0} g_{1}$, that decays subsequently to zero with a much slower rate. From the measured $\mathrm{r}_{\infty}$ value, the maximum angle $\beta$ spanned by the probe during its motion can be derived according to the relation (31):

$$
\frac{r_{\infty}}{r_{0}}=\left[\frac{\cos \beta(1+\cos \beta)}{2}\right]^{2}
$$

from which we can infer an wobbling angle of $45 \pm 1^{\circ}$ for TMR bound to both GFP 
mutants.

The addition of $\mathrm{GuHCl}$ to TMR-GFP determine an increase of both differential phase angles and demodulation ratios, as shown in Figure 4, where the upper panels report the differential phase angles and the demodulation ratios, at increasing $\mathrm{GuHCl}$ concentration from 0 to $6 \mathrm{M}$ for Mut2 and Mut2G (panel A and B, respectively). The trend of differential phase angles and demodulation ratios at increasing $\mathrm{GuHCl}$ is opposite to what expected for an increase of solution viscosity, as suggested by a measurement of the anisotropy decay of TMR-Mut2 in the presence of $4.0 \mathrm{M} \mathrm{NaCl}$, which leads to a solution whose viscosity is almost the same as that induced by $5.5 \mathrm{M}$ GuHCl. In this case in fact, both differential phase angles and demodulation ratios decrease (stars in Fig.4A), with respect to Mut2 in buffer, leading to a rotational correlation time of $14.5 \pm 0.5 \mathrm{~ns}$, in excellent agreement with the value obtained in the absence of salt once rescaled for the higher viscosity. When fitting fluorescence anisotropy data in the presence of $\mathrm{GuHCl}$ with a double exponential decay law, the longer correlation time is found to decrease versus $\mathrm{GuHCl}$ concentration for both mutants, once rescaled in order to take into account the effect of solution viscosity, as shown in the lower panels of Figure 4 (C and D for Mut2 and Mut2G, respectively). In particular, for Mut2 the correlation time decreases with a sigmoidal shape to a constant value of $5.5 \pm 0.5 \mathrm{~ns}$ at high $\mathrm{GuHCl}$ molarities, whereas for Mut2G the sigmoidal decrease is steeper and reaches a plateau value of $7.5 \pm 0.5 \mathrm{~ns}$. Interestingly, for this mutant, at even higher $\mathrm{GuHCl}$ concentrations lower values are found that can be ascribed to the presence of an additional kinetic effect, probably related to the overall GFP denaturation.

In fact, for $\mathrm{GuHCl}$ concentrations higher than 5.5 $\mathrm{M}$ for Mut2 and 4.5 $\mathrm{M}$ for Mut2G, a slow kinetic effect appears, as shown in Figure 5. For GuHCl concentrations up to $5.0 \mathrm{M}$, no time dependence is found in the differential phase angles and modulation ratios profiles for Mut2, and the derived rotational correlation time is therefore constant versus time up to several hours (Figure 5, panels A and B). A different behaviour is found at $6.0 \mathrm{M} \mathrm{GuHCl}$, where differential phase angles and demodulation ratios increase versus time: in this case the rotational correlation time decreases exponentially with a characteristic time constant of 1700 s down to values as short as 3 ns (Figure 5, panels C 
and E). Mut2G shows a similar behaviour, but lower concentrations of denaturant are needed in order to detect kinetics of the correlation time, as shown in panels $\mathrm{E}$ and $\mathrm{F}$ for 5.0 M GuHCl, where a characteristic time constant of $1900 \mathrm{~s}$ is found. This explains why the values of the rotational correlation times versus $\mathrm{GuHCl}$ concentration for Mut2G (Figure 4D) do not reach a plateau value as for Mut2: the fast kinetics at moderately high denaturant concentrations leads to a decay of the rotational correlation time on time scales shorter than the time employed to record fluorescence anisotropy data. Therefore the value found for the correlation time is affected also by this slow kinetics.

Concerning the faster rotational time in TMR-GFP FPA decays, it is found to change only slightly from 0.7 to $0.9 \pm 0.1 \mathrm{~ns}$, whereas its weight largely increases from $38 \pm 0.5 \%$ to $65 \pm 1 \%$ for Mut 2 and from $34 \pm 0.5 \%$ to $84 \pm 1 \%$ for Mut2G.

\section{DISCUSSION}

Unfolding Kinetics Detected by Chromophore Fluorescence. The unfolding kinetics curves for the two GFP mutants at $\mathrm{pH}=8.4$ can be analysed by two exponential decays as reported in the results session. Apart from an unspecific fast effect due to the $\mathrm{Cl}^{-}$ions, the relaxation time, $\tau_{\text {unf, }}$, is found to increase at increasing denaturant concentration. In particular, $\ln \left(\mathrm{k}_{\mathrm{obs}}=1 / \tau_{\mathrm{unf}}\right)$ can be fitted to a linear relation, suggesting that we are exploring the unfolding branch of the Chevron plot. The slope of the curves, which is proportional to the change in solvent exposure along the reaction (7), is similar for the two mutants, $m=\partial \ln k_{\text {obs }} / \partial[G u H C l] \cong 0.85 \pm 0.05 s^{-1} M^{-1}$, and $\mathrm{m}=1.15 \pm 0.05 \mathrm{~s}^{-}$ ${ }^{1} \mathrm{M}^{-1}$, for Mut2 and Mut2G, respectively.

The point mutation therefore has the effect of decreasing the thermodynamic stability as the pseudo-equilibrium curve for Mut2G is shifted to lower denaturant concentrations, and to speed up the unfolding process at the same $\mathrm{GuHCl}$ concentration.

This effect could either arise from a direct structural role of His148 (24) or be due to an indirect role of the histidine via the modulation of the protonation of the GFP chromophore and of the hydrogen bonding network inside the chromophore pocket $(24$, $41)$.

The Chromophore Fluorescence Does Not Reveal Changes During Unfolding. In order to unveil possible complex behaviour of the slow unfolding kinetics, spectroscopic 
techniques with different time scales have been employed. The behaviour of the chromophore fluorescence lifetime decay, that remains constant (Figure 2B) during all the kinetics seems suggesting that the quantum yield of the chromophore remains constant during the unfolding process. It is intriguing that the quantum yield does not change even after a long interaction with the denaturating agent since this implies that the rigidity of the chromophore pocket is not affected by the unfolding process unless on a time scale much shorter than the bulk experiments durations $\left(\approx 1 / \mathrm{k}_{\mathrm{obs}}\right)$ discussed above. The same conclusion can be drawn from the constancy of the rotational correlation time as derived from FPA measurements of the chromophore fluorescence (Figure 2C). In fact, no wobbling or segmental motion appears during the kinetics at any $\mathrm{GuHCl}$ concentration employed. The decrease of the fluorescence signal during the unfolding process observed in bulk solutions is then merely due to the decrease of the number of emitting molecules, and the time scale on which the bright-dark transition (coupled to the unfolding transition) occurs is much shorter than $1 / \mathrm{k}_{\mathrm{obs}}$.

An estimate of these characteristic times and of the number of fluorescent molecules can be gained by FCS. The decrease of the value of the average number of molecules $\langle\mathrm{N}\rangle$ in the excitation volume, as derived from $\mathrm{Eq}(1)$, is in excellent agreement with the analysis of the fluorescence rate decays reported in Figure 1 in the same conditions. Similar quantitative agreement has been found for the other $\mathrm{GuHCl}$ concentrations explored. Also the molecular brightness of the proteins measured at different denaturant concentrations for both the GFP mutants (right panels in Figure 3) does not change appreciably during the unfolding kinetics, in agreement with the constancy of the fluorescence lifetime and quantum yield as reported in Figure $2 \mathrm{~B}$ and suggesting again that, on the seconds time scale, the unfolding is an apparently on-off behaviour.

FCS Indicates Large $10 \mathrm{kHz}$ Fluctuations During Unfolding. Since fluorescence correlation spectroscopy probes several time decades (from microseconds to seconds), the presence of new time decay components related to the unfolding process might be revealed. In order to avoid the effect of the chromophore protonation dynamics on the ACFs, measurements have been performed at alkaline $\mathrm{pH}$, where its contribution is negligible. 
As shown in Eq. (1) and more extensively discussed in ref. (25) the photodynamic components in FCS decay exponentially. No new component is requested when fitting the data in the presence of $\mathrm{GuHCl}$ : however the fraction of the proteins that are switched off with characteristic times about $100-200 \mu$ s in the presence of $\mathrm{GuHCl}$ is found to be almost double that of the native protein. This dynamic component has been suggested to be related in the native state (25) to conformational cis-trans transitions of the chromophore (42). The present finding, of a marked overall increase of its amplitude as soon as $\mathrm{GuHCl}$ interaction starts, suggests that (i) either these isomerisation processes become more probable thanks to larger conformational fluctuations in the beta strands when $\mathrm{GuHCl}$ is present or (ii) a different mechanism is present during unfolding endowed with a characteristic time that is not separable from the cis-trans transition. The presence of flexibility in the microseconds - milliseconds time scale has been suggested by NMR studies (24), thus reinforcing the second hypothesis. The values of both the characteristic times and the associated fractions are similar for the two mutants investigated, and these values do not show time evolution during the unfolding kinetics: therefore they should be attributed to the opening of a dynamic path of the beta-barrel during the unfolding process. As confirmed by control experiments, the fraction of the dark state does not increase in the presence of $4.0 \mathrm{M} \mathrm{NaCl}$, thereby excluding any effect of chloride ions (data not shown).

TMR FPA Indicates the Occurrence of a Soft Region Involving Strand 3. Since the chromophore fluorescence does not reveal changes before the protein unfolds and then it switches off when it becomes exposed to the solvent, the fluorescence of an extrinsic probe, bound to the free cysteine 48 in strand 3 , has been monitored.

FPA decays monitored through TMR fluorescence reveal that the probe has some orientational degrees of freedom that can be followed during the unfolding. By measuring FPA at different $\mathrm{GuHCl}$ concentrations, the longer correlation time, that refers to an apparent protein rotation, decreases, whereas it is generally reported that the overall protein encumbrance is expected to show a slight increase (43) upon denaturation. The significant decrease of this apparent rotational time can be accounted for if the motion of the portion of the protein to which TMR is bound decouples from that of the whole protein and therefore the longer rotational correlation time monitors a segmental local 
motion more than an overall rotation. In particular, as shown by Lakowicz (31), when a correlation time shorter than that expected for the entire protein motion is found, a segmental motion completely free (not hindered) is present. The complete anisotropy decay can be theoretically described in this case by the sum of three exponential functions, which take into account the protein rotation $\left(\theta_{\mathrm{P}}\right)$, the segmental motion $\left(\theta_{\mathrm{S}}\right)$ and the wobbling motion of TMR at the binding site $\left(\theta_{\mathrm{w}}\right)$ : $r(t)=r_{0}\left[g_{W} \exp \left(-t / \theta_{W}\right)+g_{S} \exp \left(-t / \theta_{S}\right)+g_{P}\right] \exp \left(-t / \theta_{P}\right)$ with relative weights $\mathrm{g}_{\mathrm{W}}+\mathrm{g}_{\mathrm{S}}+\mathrm{g}_{\mathrm{P}}=1$. In our case, a weighted sum of two exponential decays, $r(t)=r_{0}\left[g_{1} \exp \left(-t / \theta_{W}^{a p p}\right)+g_{2} \exp \left(-t / \theta_{t o t}\right)\right]$ describes well the experimental data, and one can identify the relevant parameters as $\mathrm{g}_{1}=\mathrm{g}_{\mathrm{W}}$ with $\theta_{W}^{a p p}=\theta_{W} \theta_{P} /\left(\theta_{W}+\theta_{P}\right)$ and $\mathrm{g}_{2} \approx\left(\mathrm{g}_{\mathrm{P}}+\mathrm{g}_{\mathrm{S}}\right)$ with $\theta_{\text {tot }}^{-1}=\theta_{P}^{-1}+g_{S} \theta_{S}^{-1} /\left(g_{S}+g_{P}\right)$. In the limit $\mathrm{g}_{\mathrm{S}} \gg \mathrm{g}_{\mathrm{P}}$ we can estimate an upper limit for the segmental correlation time. In particular at the maximum $\mathrm{GuHCl}$ concentration explored, the longer correlation time is $5.5 \mathrm{~ns}$ with a fraction of $34 \%$ for Mut2 and $3.0 \mathrm{~ns}$ with a fraction of $57 \%$ for Mut $2 \mathrm{G}$, therefore giving markedly different limiting values of the segmental motion of $8.7 \mathrm{~ns}$ and $3.5 \mathrm{~ns}$, respectively.

Also this dynamic mode activates much earlier than the overall protein denaturation, in fact the behaviour of the FPA correlation times $\theta_{\text {tot }}$ versus $\mathrm{GuHCl}$ concentration can be fit to a sigmoidal curve for a two-state pseudo-equilibrium unfolding process leading to a midpoint denaturant concentration of $[\mathrm{GuHCl}]_{50 \%}=0.9 \pm$ $0.2 \mathrm{M}$ and $[\mathrm{GuHCl}]_{50 \%}=0.4 \pm 0.1$ for Mut2 and Mut2G, respectively. These values are much lower than those found when monitoring the chromophore fluorescence, (Fig1A) (7, 32, Pioselli et al., manuscript in preparation). An effect of TMR bound to GFP can be ruled out by detecting the circular dichroism signal at different $\mathrm{GuHCl}$ concentrations, showing that the secondary structure and the protein stability are not substantially affected by the TMR presence.

These findings agree with the increase of the TMR wobbling weight leading to larger angles spanned by the probe during its motion at the binding site (from $45^{\circ}$ to $70^{\circ}$ for Mut2 and up to $85^{\circ}$ for Mut2G), a picture consistent with a progressive structure loosening which is more pronounced for Mut2G (24). The process of loosening of strand 
3 is faster than the global GFP unfolding monitored by the chromophore, but its kinetics also contains a very slow phase (Figure 5), two or threefold slower than the kinetic detected by the chromophore fluorescence decrease, suggesting that even after its fluorescence has been quenched by the solvent, local regions of the protein continue to experience conformational changes.

\section{SUMMARY}

In this report we characterize the unfolding kinetics of two GFP mutants on a wide time range, from ns to seconds, exploiting spectroscopic techniques with different dynamic ranges.

The single point mutation introduced in Mut2 to yield Mut2G, in addition to the large effect found in the chromophore protonation dynamics resulting in pronounced shift in the chromophore $\mathrm{pK}$, affects the thermodynamic stability of the protein, and also its kinetics leading, at alkaline $\mathrm{pH}$ where protonation is negligible, to a much faster unfolding process. The looser structure of Mut $2 \mathrm{G}$ is evident both in the larger angle spanned by TMR probe and in the larger contribution of the segmental motion as detected by FPA. This peculiarity of Mut $2 \mathrm{G}$ could be exploited in the rational design of mutants to coupling improved photochromic properties with dynamical structural flexibility.

Concerning the unfolding process of both mutants, the use of an extrinsic fluorescent probe has allowed to point out that the region around strand 3 of the beta barrel structure looses its rigidity, at least at the level of the hinges between the beta strands, far before the chromophore pocket, that is responsible for the rigidity of the chromophore and therefore for its high fluorescence quantum yield. The characteristic time of the hinge motions falls in the region of nanoseconds. In addition to this motions revealed by the probe segmental motions, a far slower conformational change is also detected in this region that starts to loosen up at very low $\mathrm{GuHCl}$ concentrations and continue to increase the decoupling from the whole protein even after the beta-barrel has ceased to prevent the exposure of the chromophore to the solvent, therefore on time scales of thousands of seconds.

On the other side, the conformational fluctuations actually occurring around the chromophore are likely to occur on a time scale of 100-200 $\mu \mathrm{s}$, as revealed by the 
substantial increase of this relaxation component in the fluorescence autocorrelation curves indicating that an increase in the fraction of protein falling in a dark state. Both the hinge (ns) and isomerization $(\approx 100 \mu \mathrm{s})$ processes occur during the whole unfolding kinetics.

This very articulate scenario, encompassing equilibrium and kinetic intermediates, or different time scales depending on the reporter chosen, suggests that GFP unfolding is a very complex process also from the kinetic point of view, accomplishing the picture of a series of potential barriers that have to be overcome in order to unfold, as recently depicted $(12,18,22)$.

Acknowledgments. This research has been partially funded by a PRIN Project 2008 (to M.C. and S.B.). 


\section{REFERENCES}

1. R. Y. Tsien, Constructing and exploiting the fluorescent protein paintbox (Nobel Lecture), Angew. Chem. Int. Ed. Engl. 48, (2009) 5612-5626.

2. R. Rizzuto, M. Brini, P. Pizzo, M. Murgia, T. Pozzan, Chimeric green fluorescent protein as a tool for visualizing subcellular organelles in living cells, Curr. Biol. 5, (1995) 635-642.

3. S.T. Hsu, G. Blaser, S.E. Jackson, The folding, stability and conformational dynamics of beta-barrel fluorescent proteins, Chem. Soc. Rev. 38, (2009) 29512965.

4. R.N. Day, M.W. Davidson, The fluorescent protein palette: tools for cellular imaging, Chem. Soc. Rev. 38, (2009) 2887-2921.

5. S.W. Hell, Towards fluorescence nanoscopy, Nature Biotech. 21, (2003) 13471355.

6. M. Andresen, A. C. Stiel, J. Fölling, D. Wenzel, A. Schönle, A. Egner, C. Eggeling, S. W. Hell, S. Jakobs, Photoswitchable fluorescent proteins enable monochromatic multilabel imaging and dual color fluorescence nanoscopy, Nature Biotech. 26, (2008) 1035-1040.

7. B. Campanini, S. Bologna, F. Cannone, G. Chirico, A. Mozzarelli, S. Bettati, Unfolding of Green Fluorescent Protein mut2 in wet nanoporous silica gels, Protein Sci. 14, (2005) 1125-1133.

8. H. Fukuda, M. Arai, K. Kuwajima, Folding of green fluorescent protein and the Cycle3 mutant, Biochemistry. 39, (2000) 12025-12032.

9. R. Battistutta, A. Negro, G. Zanotti, Crystal structure and refolding properties of the mutant F99S/M153T/V163A of the Green Fluorescent Protein, ProteinsStructure Function and Genetics 41, (2000) 429-437.

10. S. Enoki, K. Saeki, K. Maki, K. Kuwajima, Acid denaturation and refolding of green fluorescent protein, Biochemistry. 43, (2004) 14238-14248.

11. S. Enoki, K. Maki, T. Inobe, K. Takahashi, K. Kamagata, T. Oroguchi, H. Nakatani, K. Tomoyori, K. Kuwajima, The equilibrium unfolding intermediate observed at $\mathrm{pH} 4$ and its relationship with the kinetic folding intermediates in green fluorescent protein, J. Mol. Biol. 361, (2006) 969-982.

12. J.R. Huang, T. D. Craggs, J. Christodoulou, S.E. Jackson, Stable intermediate states and high energy barriers in the unfolding of GFP, J. Mol. Biol. 370, (2007) 356-371.

13. S.E. Jackson, T. D. Craggs, J. R. Huang, J. R. Understanding the folding of GFP using biophysical techniques, Expert Rev. Proteomics 3, (2006) 545-559.

14. O.V. Stepanenko, V. V. Verkhusha, V. I. Kazakov, M. M. Shavlovsky, I. M. Kuznetsova, V. N. Uversky, K. K. Turoverov, Comparative studies on the structure and stability of fluorescent proteins EGFP, zFP506, mRFP1, "dimer2", and DsRed1, Biochemistry. 43, (2004) 14913-14923.

15. O.V. Stepanenko, V. V. Verkhusha, I. M. Kuznetsova, V. N. Uversky, K. K. Turoverov, Fluorescent proteins as biomarkers and biosensors: throwing color lights on molecular and cellular processes, Curr. Protein Pept. Sci. 9, (2008) 338369. 
16. J.B. Xie, J. M. Zhou, Trigger factor assisted folding of green fluorescent protein, Biochemistry. 47, (2008) 348-357.

17. S.T. Hsu, G. Blaser, C. Behrens, L.D. Cabrita, C. M. Dobson, S. E. Jackson, Folding study of Venus reveals a strong ion dependence of its yellow fluorescence under mildly acidic conditions, J. Biol. Chem. 285, (2010) 4859-4869.

18. B.T. Andrews, A. R. Schoenfish, M. Roy, G. Waldo, P.A. Jennings, The rough energy landscape of superfolder GFP is linked to the chromophore, J. Mol. Biol. 373, (2007) 476-490.

19. A. Orte, T.D. Craggs, S. S. White, S. E. Jackson, D. Klenerman, Evidence of an intermediate and parallel pathways in protein unfolding from single-molecule fluorescence, J. Am. Chem. Soc. 130, (2008) 7898-7907.

20. M. Bertz, A. Kunfermann, M. Rief, Navigating the folding energy landscape of green fluorescent protein, Angew. Chem. Int. Ed. Engl. 47, (2008) 8192-8195.

21. B.T. Andrews, S. Gosavi, J.M. Finke, J. N. Onuchic, P. A. Jennings, The dualbasin landscape in GFP folding, Proc. Natl. Acad. Sci. U. S. A. 105, (2008) 12283-12288.

22. M. Mickler, R.I. Dima, H. Dietz, C. Hyeon, D. Thirumalai, M. Rief, Revealing the bifurcation in the unfolding pathways of GFP by using single-molecule experiments and simulations, Proc. Natl. Acad. Sci. U. S. A. 104, (2007) 2026820273.

23. G. Baldini, F. Cannone, G. Chirico, M. Collini, B. Campanini, S. Bettati, A. Mozzarelli, Evidence of discrete substates and unfolding pathways in green fluorescent protein, Biophys. J. 92, (2007) 1724-1731.

24. M.H.J. Seifert, J. Georgescu, D. Ksiazek, P. Smialowski, T. Rehm, B. Steipe, T.A. Holak, Backbone dynamics of green fluorescent protein and the effect of histidine 148 substitution, Biochemistry. 42, (2003) 2500-2512.

25. C. Bosisio, V. Quercioli, M. Collini, L. D'Alfonso, G. Baldini, S. Bettati, B. Campanini, S. Raboni, G. Chirico, Protonation and conformational dynamics of GFP mutants by two-photon excitation fluorescence correlation spectroscopy, $J$. Phys. Chem. B 112, (2008) 8806-8814.

26. B.P. Cormack, R. H. Valdivia, S. Falkow, FACS-optimized mutants of the green fluorescent protein (GFP), Gene 173, (1996) 33-38.

27. G. Chirico, F. Cannone, S. Beretta, A. Diaspro, B. Campanini, S. Bettati, R. Ruotolo, A. Mozzarelli, Dynamics of green fluorescent protein mutant2 in solution, on spin-coated glasses, and encapsulated in wet silica gels, Protein Sci. 11, (2002) 1152-1161.

28. S. Abbruzzetti, E. Grandi, C. Viappiani, S. Bologna, B. Campanini, S. Raboni, S. Bettati, A. Mozzarelli, Kinetics of acid-induced spectral changes in the GFPmut2 chromophore, J. Am. Chem. Soc. 127, (2005) 626-635.

29. G. Malengo, R. Milani, F. Cannone, S. Krol, A. Diaspro, G. Chirico, High sensitivity optical microscope for single molecule spectroscopy studies, Rev. Sci. Instrum. 75, (2004) 2746-2751.

30. M. Collini, G. Chirico, G. Baldini, M.E. Bianchi, Conformation of short DNA fragments by modulated fluorescence polarization anisotropy, Biopolymers 36 , (1995) 211-225. 
31. Lakowicz, J. R. Principles of Fluorescence Spectroscopy, (2nd ed., Kluver Academic/Plenum Publishers, New York, 1999).

32. F. Cannone, S. Bologna, B. Campanini, A. Diaspro, S. Bettati, A. Mozzarelli, G. Chirico, Tracking unfolding and refolding of single GFPmut2 molecules, Biophys. J. 89, (2005) 2033-2045.

33. R. Bizzarri, C. Arcangeli, D. Arosio, F. Ricci, P. Faraci, F. Cardarelli, F. Beltram, Development of a novel GFP-based ratiometric excitation and emission $\mathrm{pH}$ indicator for intracellular studies, Biophys. J. 90, (2006) 3300-331.

34. R.M. Wachter, Remington S.J. Sensitivity of the yellow variant of green fluorescent protein to halides and nitrate, Curr. Biol. 9, (1999) R628-R629.

35 D. Arosio, Garau G., Ricci F., Marchetti L., Bizzarri R., Nifosì R., Beltram F., Spectroscopic and Structural Study of Proton and Halide Ion Cooperative Binding to GFP, Biophys. J. 93, (2007) 232-244.

36. L. D'Alfonso, M. Collini, G. Baldini, Evidence of heterogeneous 1anilinonaphthalene-8-sulfonate binding to beta-lactoglobulin from fluorescence spectroscopy, Biochim. Biophys. Acta 1432, (1999) 194-202.

37. Doi M. Edwards S.F. The theory of polymer dynamics (Oxford University Press, USA, 1986)

38. A.A. Heikal, S. T. Hess, W. W. Webb, Multiphoton molecular spectroscopy and excited-state dynamics of enhanced green fluorescent protein (EGFP): acid-base specificity, Chemical Physics 274, (2001) 37-55.

39. K. Suhling, D.M. Davis, D. Phillips, The Influence of Solvent Viscosity on the Fluorescence Decay and Time-Resolved Anisotropy of Green Fluorescent Protein, Journal of Fluorescence 12, (2002) 91-95.

40. A. Volkmer, V. Subramaniam, D.J. Birch, T. M. Jovin, One- and two-photon excited fluorescence lifetimes and anisotropy decays of green fluorescent proteins, Biophys. J. 78, (2000) 1589-1598.

41. S.J. Remington, Fluorescent proteins: maturation, photochemistry and photophysics, Curr. Opin. Struct. Biol. 16, (2006) 714-721.

42. S. Luin, V. Voliani, G. Lanza, R. Bizzarri, P. Amat, V. Tozzini, M. Serresi, F. Beltram, Raman study of chromophore states in photochromic fluorescent proteins, J. Am. Chem. Soc. 131, (2009) 96-103.

43. K. Chattopadhyay, S. Saffarian, E.L. Elson, C. Frieden, Measurement of microsecond dynamic motion in the intestinal fatty acid binding protein by using fluorescence correlation spectroscopy, Proc. Natl. Acad. Sci. U. S. A. 99, (2002) 14171-14176. 
Table 1. Fluorescence anisotropy decay parameters for GFP mutants.

$\begin{array}{lcccccc}\text { Mutant } & \theta_{1}(\mathrm{~ns}) & \mathrm{g}_{1}(\%) & \theta_{2}(\mathrm{~ns}) & \mathrm{g}_{2}(\%) & \mathrm{r}_{0} & \mathrm{R}_{\mathrm{eff}}(\mathrm{nm}) \\ \text { Mut2 } & 13.6 \pm 0.1 & 100 & - & - & 0.390 \pm 0.001 & 2.37 \pm 0.01 \\ \text { Mut2G } & 14.4 \pm 0.2 & 100 & - & - & 0.390 \pm 0.001 & 2.43 \pm 0.02 \\ \text { TMR-Mut2 } & 15.0 \pm 0.5 & 38 \pm 2 & 0.7 \pm 0.2 & 62 \pm 2 & 0.300 \pm 0.002 & 2.45 \pm 0.03 \\ & & & & & & \\ \text { TMR-Mut2G } & 15.4 \pm 0.5 & 34 \pm 2 & 0.7 \pm 0.2 & 66 \pm 3 & 0.324 \pm 0.005 & 2.47 \pm 0.03\end{array}$

FPA decay parameters for both GFP mutants at $\mathrm{pH}=8.4$ in the absence of $\mathrm{GuHCl}$. The first two rows refer to FPA decay acquired through the chromophore fluorescence $(515 / 30 \mathrm{~nm})$, the last two rows refer to FPA decay acquired through TMR fluorescence $(560 / 40 \mathrm{~nm})$. 


\section{FIGURE CAPTIONS.}

Figure 1. Panel A. Pseudo-equilibrium unfolding curves shown as the denatured fraction of proteins versus $\mathrm{GuHCl}$ concentration for Mut2 (full circles) and Mut2G (open triangles). A sigmoidal fit is superimposed to the data according to the expression $f_{D}=\frac{\exp \left(-m\left([G u H C l]_{\text {mid }}-[G u H C l]\right) / R T\right)}{1+\exp \left(-[G u H C l]_{\text {mid }}-[G u H C l] / R T\right)}$ Panel B. Natural logarithm of the effective unfolding constant, $\mathrm{k}_{\mathrm{obs}}$, as a function of $\mathrm{GuHCl}$ concentration for Mut2 (full circles) and Mut2G (open triangles). Panel C. Mut2 normalized fluorescence signal at $510 \mathrm{~nm}$ at $\mathrm{pH}=8.4$ versus time at different denaturant concentrations: $5.0 \mathrm{M} \mathrm{GuHCl}$ (squares), $5.2 \mathrm{M}$ $\mathrm{GuHCl}$ (circles), $5.5 \mathrm{M} \mathrm{GuHCl}$ (up triangles), $6.0 \mathrm{M} \mathrm{GuHCl}$ (down triangles). Panel D. Mut2G normalized fluorescence signal at $510 \mathrm{~nm}$ at $\mathrm{pH}=8.4$ versus time at different denaturant concentrations: $4.2 \mathrm{M} \mathrm{GuHCl}$ (squares), $4.5 \mathrm{M} \mathrm{GuHCl}$ (circles), $5.0 \mathrm{M} \mathrm{GuHCl}$ (up triangles), $5.2 \mathrm{M} \mathrm{GuHCl}$ (down triangles). The continuous lines represent the two exponential fit of the data in both panels.

Figure 2. Panel A: ratio of the fluorescence signal at $510 \mathrm{~nm}$ in the presence and in the absence of $\mathrm{NaCl}$ versus time at $\mathrm{pH} 8.4$ for Mut2 (full circles) and Mut2G (open triangles). Panel B: ratio of the fluorescence lifetime in the presence and in the absence of $\mathrm{GuHCl}$ versus time, at $\mathrm{pH} 8.4$ for Mut2 at $5.5 \mathrm{M} \mathrm{GuHCl}$ (closed circles) and Mut2G at $4.5 \mathrm{M} \mathrm{GuHCl}$ (open triangles). Panel C: ratio of the rotational correlation time probed by the chromophore fluorescence in the absence and in the presence of $\mathrm{GuHCl}$ in the same conditions as panel B versus time. Panel D: rotational correlation time probed by the chromophore fluorescence, rescaled according to the solution viscosity, at increasing $\mathrm{GuHCl}$ concentration. The symbols are the same as in panel B.

Figure 3. Panel A: fluorescence ACFs at increasing time after the addition of $5.2 \mathrm{M}$ $\mathrm{GuHCl}$ for Mut2 at $\mathrm{pH}=8.4$. The continuous lines are the fit according to Eq. (1). The curve with full symbols represents the ACF in the absence of $\mathrm{GuHCl}$. Panel B: fluorescence ACFs at increasing time after the addition of $4.4 \mathrm{M} \mathrm{GuHCl}$ for Mut2 $\mathrm{G}$ at $\mathrm{pH}=8.4$. The continuous lines are best fit of the data according to Eq. (1). The curve with 
full symbols represents the ACF in the absence of $\mathrm{GuHCl}$. Inset in panel B: Fraction of protein molecules in the dark state associated to the photodynamic component as discussed in the text for Mut2 (circles) and Mut2G (triangles) in same conditions as in panel A and B; in full symbols are the values in the absence of denaturant. Panel C and D: normalized number of proteins in the excitation volume versus time as derived from the $\mathrm{G}(0)$ values obtained from ACFs fitting to Eq.(1) for Mut2 (circles) and for Mut2G (triangles) in the same conditions as in panel A and B. The insets in both panels show the molecular brightness, in $\mathrm{KHz}$, for Mut2 (circles) and for Mut2G (triangles) versus time in the same conditions as in panel A and B.

Figure 4. Upper panels: differential phase angles (left axis, full symbols) and demodulation ratios (right axis, open symbols) versus modulation frequency at increasing $\mathrm{GuHCl}$ concentration from 0 to $6.0 \mathrm{M}$ at $\mathrm{pH} 8.4$ for Mut2 (panel A, circles) and Mut2G (panel B, triangles). In panel A, the stars refer to the addition of $4.0 \mathrm{M} \mathrm{NaCl}$ to Mut2. Lower panels: longer relaxation time of the FPA decay versus denaturant concentration as derived from a two exponential data fitting for Mut2 (panel C, circles) and Mut2G (panel D, triangles). The continuous lines represent a sigmoidal fit as discussed in the text. Inset in panel B: residue molar ellipticity $\left(\mathrm{deg} \mathrm{cm}^{2} \mathrm{dmol}^{-1}\right)$ of TMR-Mut2 at different denaturant concentrations: from bottom to top, $0 \mathrm{M}, 1 \mathrm{M}, 2 \mathrm{M} 4 \mathrm{M} \mathrm{GuHCl}$.

Figure 5. Panel A: time-dependence of the rotational correlation times of Mut2 in $5.0 \mathrm{M}$ $\mathrm{GuHCl}$, as derived by fitting the raw data shown in panel B (differential phase angles, left axis, full circles, and modulation ratios, right axis, open circles) versus modulation frequency at different times. Panel C: rotational correlation times for Mut2 in $6.0 \mathrm{M}$ $\mathrm{GuHCl}$ versus time. The raw data are shown in panel D (differential phase angles, left axis, full circles, and modulation ratios, right axis, open circles) versus modulation frequency. Panel E: rotational correlation times for Mut2G in 5.0 M GuHCl versus time. The raw data are shown in panel $\mathrm{F}$ (differential phase angles, left axis, full triangles, and modulation ratios, right axis, open triangles) versus modulation frequency. 
1. We have shown that the point mutation H148G speeds up GFP unfolding by chemical agents.

2. Fluorescence correlation spectroscopy shuows increased photodynamics during unfolding in the 100-200 $\mu$ s range

3. Tetramethylrhodamine bound to cys 48 of GFP mutants reveals a soft region that starts unfolding at lower denaturant concentrations than that near the chromophore. 
GuHCl (M)

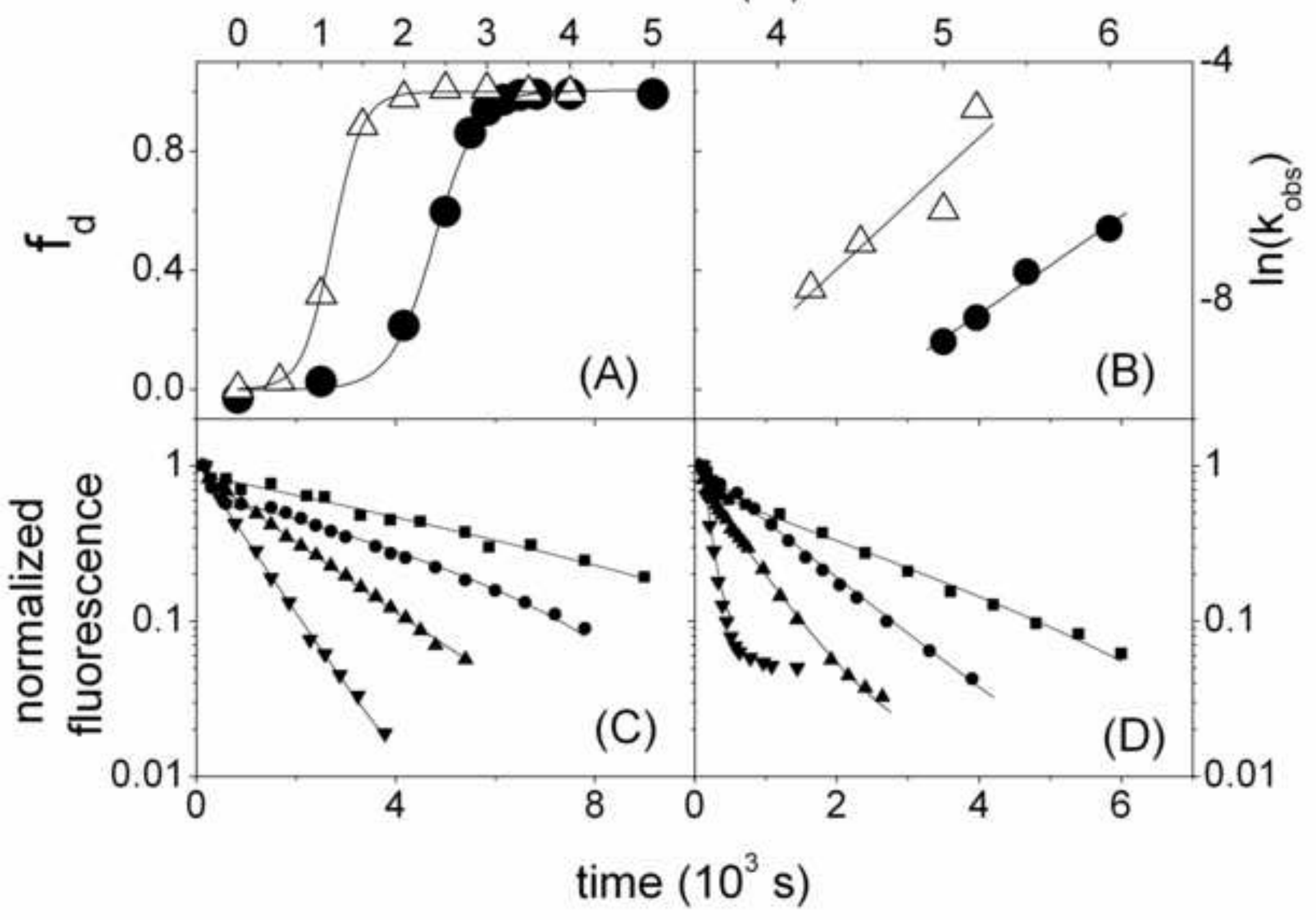



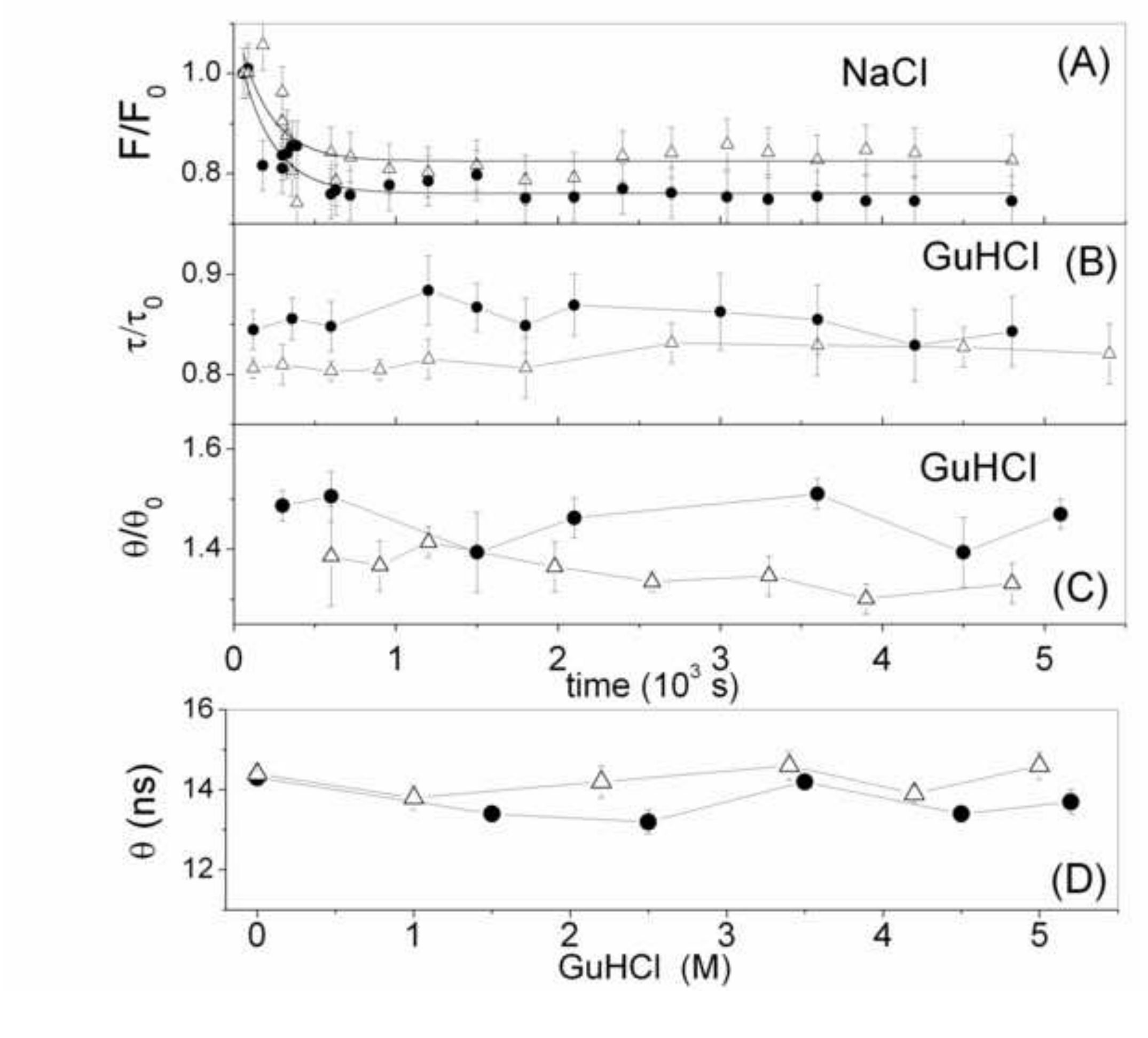

ACCEPTED MANUSCRIPT

.

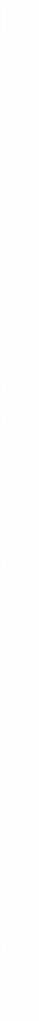




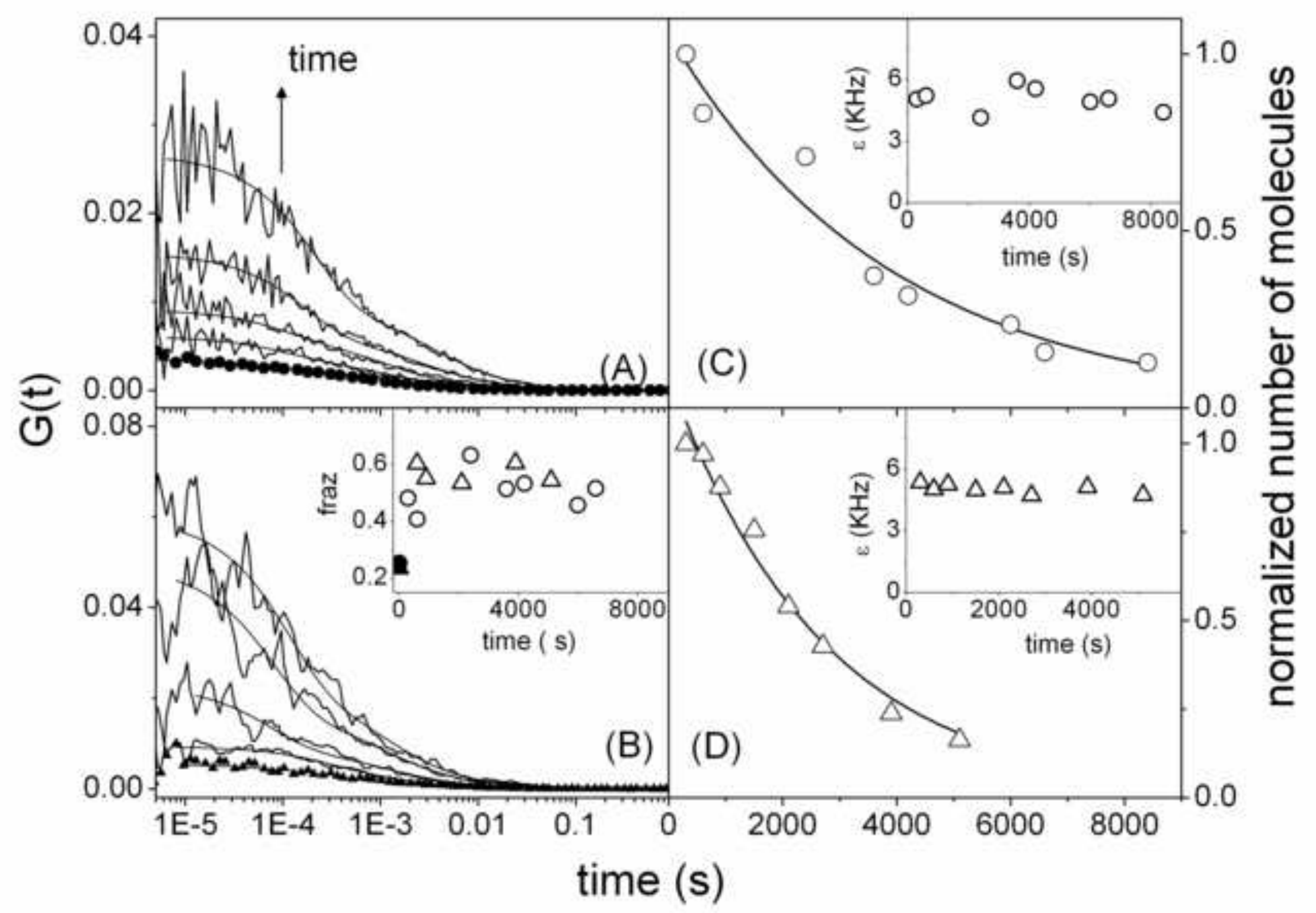




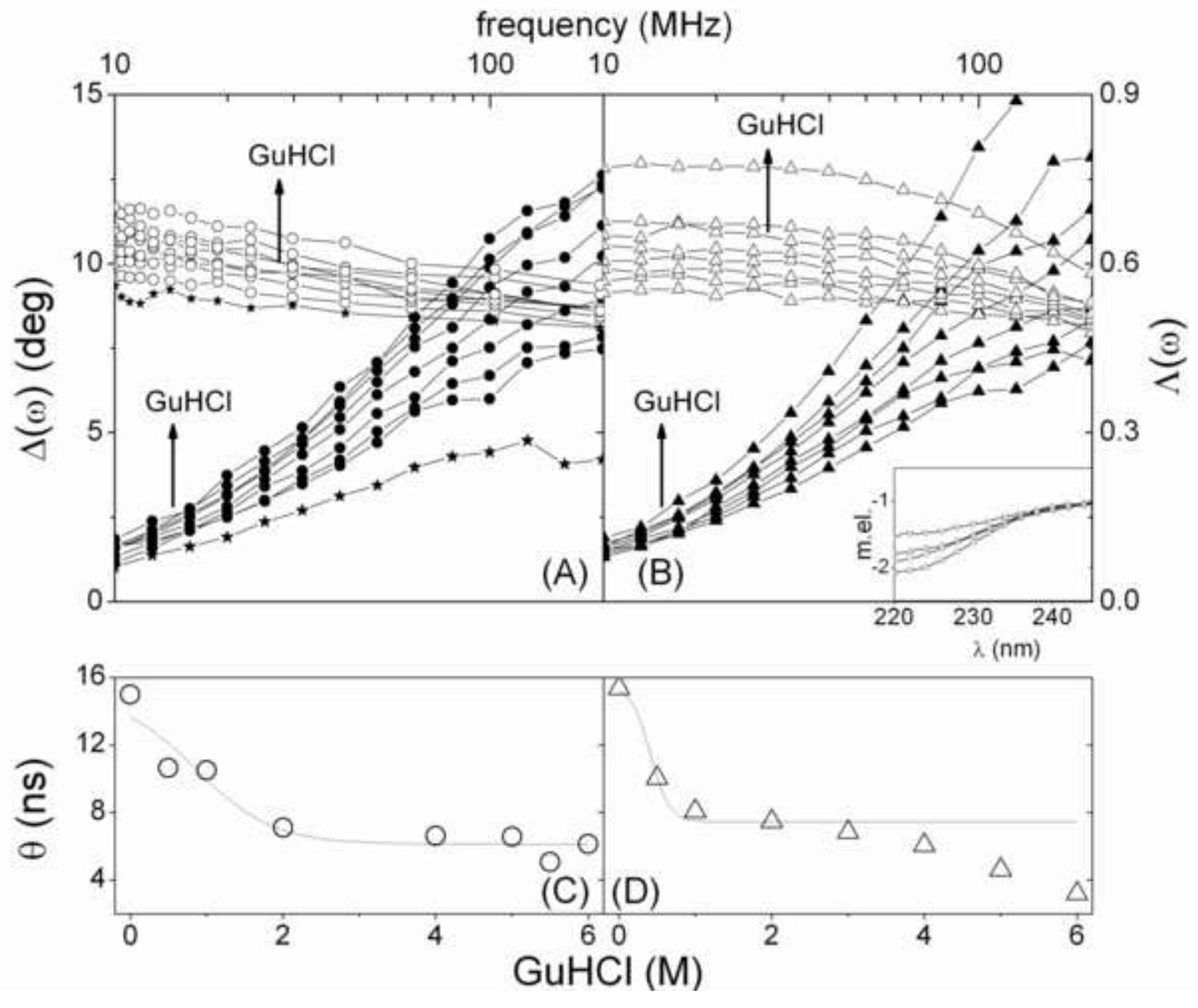

frequency $(\mathrm{MHz})$ 

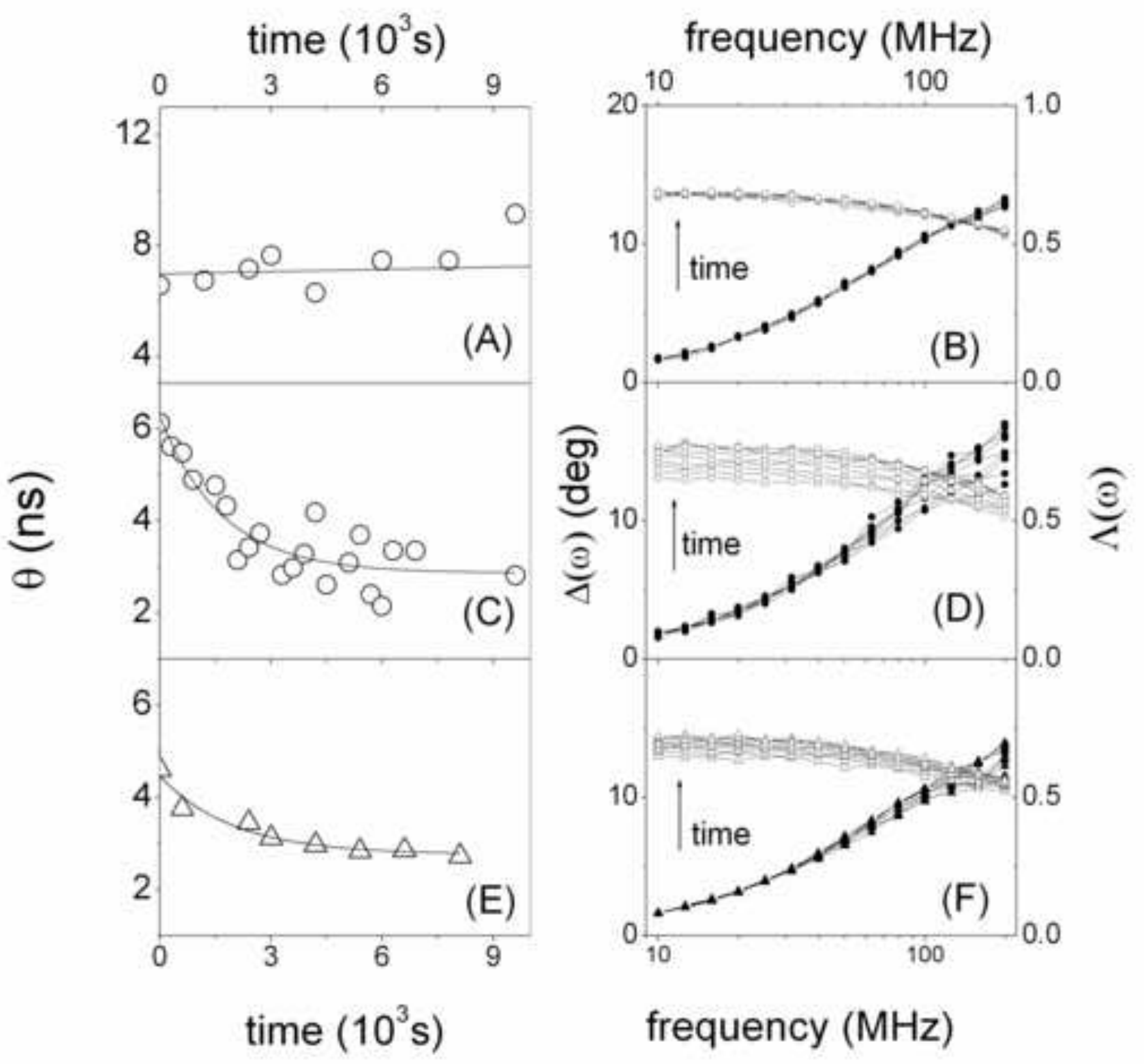


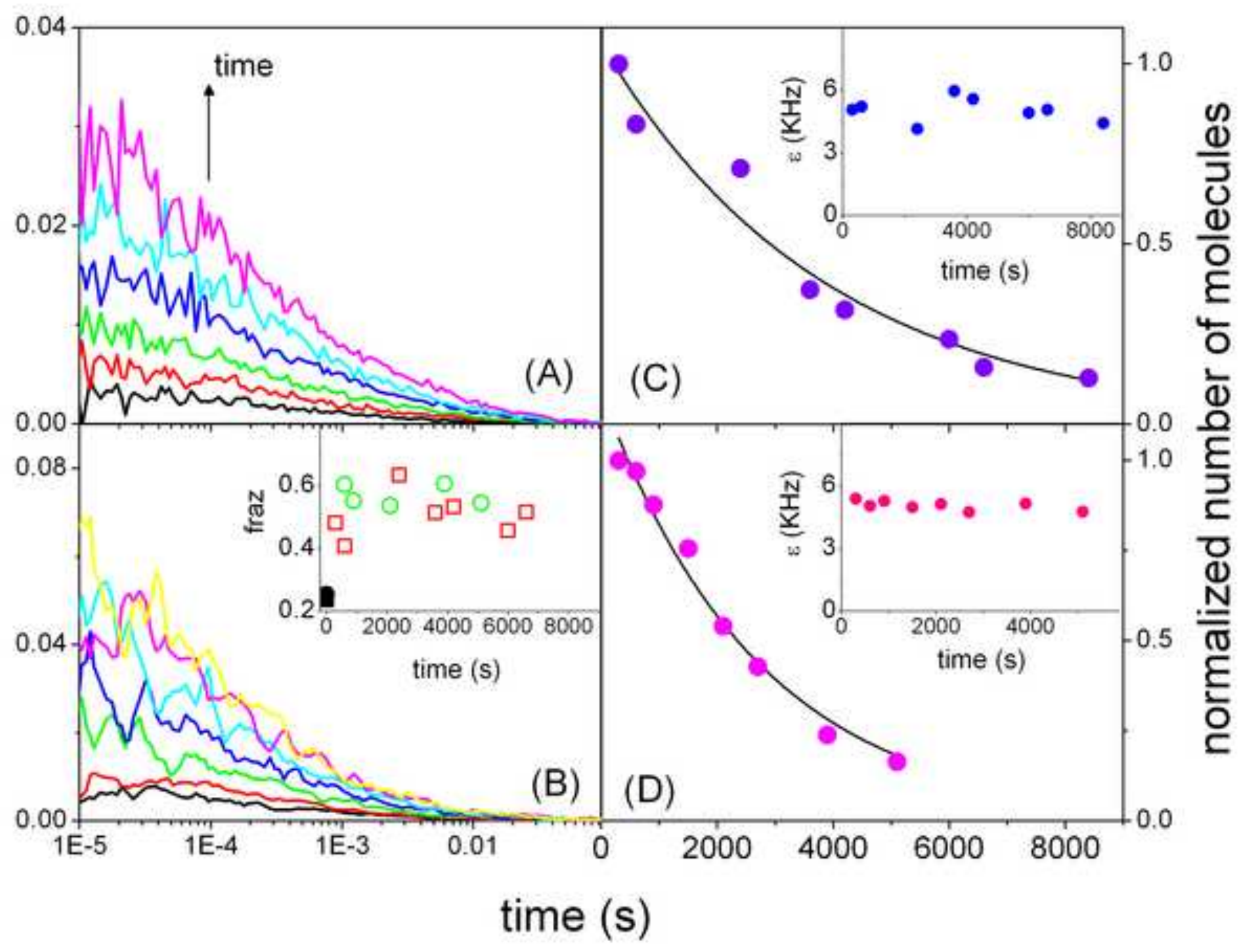

